\title{
Effect of Different Injection Rates and Doses of Contrast Medium on the Image Quality of Computed Tomographic Angiography in African Grey Parrots (Psittacus Erithacus)
}

\section{Wen-Lin Wang}

National Taiwan University

Fang-Lun Chang

National Taiwan University Veterinary Hospital

Pin-Huan Yu ( $\sim$ pinhuan@ntu.edu.tw )

National Taiwan University

\section{Research Article}

Keywords: African grey parrots, atherosclerosis, computed tomography angiography, contrast medium, image quality

Posted Date: September 22nd, 2021

DOI: https://doi.org/10.21203/rs.3.rs-898289/v1

License: @ (i) This work is licensed under a Creative Commons Attribution 4.0 International License. Read Full License 


\section{Abstract \\ Background}

Atherosclerosis is a common cardiovascular disease in parrots but the antemortem diagnosis is challenging. In human medicine, computed tomography angiography has been used widely for the diagnosis of atherosclerosis. By adjusting the injection conditions (injection rate and total dose of contrast medium [CM]), the image quality can be improved. To test the effects of different CM injection conditions on the image quality of major arteries, 10 African grey parrots (Psittacus erithacus) were used. Three injection rates $(0.3,0.4,0.5 \mathrm{~mL} / \mathrm{s})$ and three $\mathrm{CM}$ doses $(740,370,222 \mathrm{mg}$ of iodine/bird) were tested while the other variables of the studies were fixed.

\section{Results}

We found no difference of the measured diameter, attenuation, image noise, signal-to-noise ratio, or contrast-to-noise ratio of the six major arteries ( $p>0.05)$ among these three $\mathrm{CM}$ injection rates. However, these parameters showed significant differences among the three $\mathrm{CM}$ injection doses. Furthermore, with increasing $\mathrm{CM}$ injection rates, the prevalence of heterogeneity decreased.

\section{Conclusion}

We recommend a combination of $370 \mathrm{mg}$ of iodine/bird with $0.4 \mathrm{~mL} / \mathrm{s}$ for clinical use to achieve better image quality for CTA.

\section{Background}

Atherosclerosis is a common finding upon postmortem examination in companion Psittaciformes [1]. The prevalence of atherosclerosis in parrots has been found to be 1.9-91.8\% on postmortem examinations [2-9]. Among these species, African grey parrots (Psittacus erithacus; AGPs) and Hispaniolan Amazon parrots (Amazona ventralis) have the highest prevalence.

Diagnosing atherosclerosis antemortem in birds is difficult [10-16]. A tentative diagnosis can be made based on clinical presentation and risk factors for atherosclerosis, but a highly presumptive diagnosis might rely primarily on diagnostic imaging [17-19]. Computed tomography angiography (CTA) is a valuable tool and may become a "gold standard" for diagnosing avian atherosclerosis.

CTA guidelines in human medicine (HM) are well-documented [20,21]. Some factors can affect vessel enhancement and image quality, and can be divided into three categories: patient, CT, and contrast medium (CM) [22]. With different patient conditions or target organs, practitioners can adjust the factors accordingly to enhance image quality. Little work has been done on how these factors influence image conditions in avian patients.

A study of nine AGPs found that it was easier to interpret the blood-vessel diameter post-contrast at the body window [23]. Another study of nine AGPs employed three timings for saline flush during $\mathrm{CM}$ infusion and found that saline flush after CM infusion elicited the most reliable measurements of major arteries owing to the lower heterogeneity of contrast distribution [24]. The injection rate (IR) effect on contrast enhancement has been studied in HM. With a fixed total amount of CM, a faster IR can increase the blood-delivery rate and total amount of CM delivered, which enhances blood-vessel attenuation [22]. The problem of heterogeneity can be overcome by increasing the IR of CM.

As for injection doses, in a study of Hispaniolan Amazon parrots, high intra-observer agreement was obtained using $720 \mathrm{mg}$ of iodine/kg [25]. In a study of AGPs, $1682 \mathrm{mg}$ of iodine/kg was used [23]. The latter study achieved optimal image quality with "moderate"-to-"strong" intra-observer agreement and interobserver agreement, but attenuation might have been too high (> $500 \mathrm{HU}$ [Hounsfield unit]) for left and right brachiocephalic trunks and the ascending aorta (AA). Furthermore, CM may have caused some side-effects (e.g., nephrotoxicity, allergic reaction) [26]. Therefore, our aim was to decrease the dose of CM.

We report the effect of different IRs and doses of CM on image quality in avian CTA. Subsequently, the attenuation of major arteries under different conditions of CM was compared. We hypothesized that: (a) with an increased IR of CM, the heterogeneity of CM distribution would be limited and elicit better image quality; (b) with a reduced dose of $\mathrm{CM}$, attenuation of major arteries would decrease.

\section{Results}

\section{Animal condition and CTA}

All AGPs were healthy based on the examinations mentioned above before conducting CTA.

During recovery following an injection protocol of $0.4 \mathrm{~mL} / \mathrm{s}$ with $222 \mathrm{mg}$ of iodine/bird, mild crackles were detected at the left flank via auscultation in one of 10 AGPs. Two of the 10 AGPs regurgitated during recovery following an injection protocol of $0.5 \mathrm{~mL} / \mathrm{s}$ with 222 mg of iodine/bird. Other adverse effects were not observed in other $\mathrm{CM}$ injection protocols. AGPs recovered uneventfully $<1 \mathrm{~h}$ after anesthesia.

Among the 90 studies, there were two imaging failures in the same parrot owing to $\mathrm{CM}$ leakage from the intravenous catheter. One was during the injection protocol of $0.5 \mathrm{~mL} / \mathrm{s}$ with $740 \mathrm{mg}$ of iodine/bird, and the other was during the injection protocol of $0.5 \mathrm{~mL} / \mathrm{s}$ with $222 \mathrm{mg}$ of iodine/bird.

Objective (quantitative) image quality 
A decrease in the $\mathrm{CM}$ dose led to a progressive decrease in attenuation (Figure 1). Mean vascular attenuation was significantly different between the three groups of $740 \mathrm{mg}$ of iodine/bird and three groups of $222 \mathrm{mg}$ of iodine/bird in all six major arteries $(p<0.0006)$. There was no significant difference between attenuation measured with different IRs.

A decrease in the $\mathrm{CM}$ dose also led to a progressive decrease in image noise (Figure 2). In the AA, there was a significant and progressive decrease in image noise between the three groups of $740 \mathrm{mg}$ of iodine/bird and three groups of $370 \mathrm{mg}$ of iodine/bird ( $p<0.0001)$; and between the three groups of $740 \mathrm{mg}$ of iodine/bird and three groups of $222 \mathrm{mg}$ of iodine/bird $(p<0.0001)$. In the other five major arteries, image noise was significantly higher in the three groups of $740 \mathrm{mg}$ of iodine/bird compared with that in the group of $222 \mathrm{mg}$ of iodine/bird $(p<0.0005)$. There was no significant difference in image noise among the remaining groups.

A decrease in the CM dose led to progressive increases in signal-to-noise ratio (SNR) and contrast-to-noise ratio (CNR) (Figure 3). Within the nine study groups, SNR and CNR were lower in three groups of $740 \mathrm{mg}$ of iodine/bird than in three groups of $222 \mathrm{mg}$ of iodine/bird. The greatest SNR and CNR were seen in the $222 \mathrm{mg}$ of iodine/bird groups. Differences in SNR and CNR were not significant among the three IRs tested.

Arterial diameters were larger in higher dose groups than those of lower dose groups (Figure 4). Measured diameters of the six arteries were significantly larger in the three groups of $740 \mathrm{mg}$ of iodine/bird compared with those of the three groups of $222 \mathrm{mg}$ of iodine/bird $(p<0.0012)$. This phenomenon was more obvious in vessels with wider diameters (i.e., left brachiocephalic trunks, right brachiocephalic trunks, and AA). Arterial diameters were similar among the groups with the same dose but different IRs.

Two-way ANOVA revealed that the injection dose significantly affected the results of these five objective parameters in the six arteries $(p<0.0001)$.

Inter-observer agreement for attenuation and diameter measurement was "strong" to "very strong" in the 370 mg of iodine/bird groups and 222 mg of iodine/bird groups, compared with that in the $740 \mathrm{mg}$ of iodine/bird groups, which was "moderate" to "strong" (Table 1). For intra-observer agreement (reproducibility), in most cases, $\rho \geq 0.8$, indicating a "very strong" correlation, with very few exceptions for small arteries (i.e., right pulmonary aorta and abdominal aorta) in AGPs undergoing protocols of $740 \mathrm{mg}$ of iodine/bird and $222 \mathrm{mg}$ of iodine/bird ( $\rho<0.6$; Table 2). There was no significant difference in intra-observer and inter-observer agreements among these nine groups $(p>0.05)$.

\section{Subjective (qualitative) image quality}

Scoring results for subjective image quality integrated from two observers are listed in Table 3. Subjective image quality at the six arteries was significantly lower among groups administered $370 \mathrm{mg}$ of iodine/bird versus groups given $740 \mathrm{mg}$ of iodine/bird and $222 \mathrm{mg}$ of iodine/bird ( $p<0.0007$ ). Comparison between groups according to different CM doses led to both observers noting a significant degradation of image quality in groups with $222 \mathrm{mg}$ of iodine/bird versus other groups. There were more instances of image quality being scored "non-diagnostic" in the $222 \mathrm{mg}$ of iodine/bird with $0.4 \mathrm{~mL} / \mathrm{s}$ group and $222 \mathrm{mg}$ of iodine/bird with $0.5 \mathrm{~mL} / \mathrm{s}$ group. The scores of these two groups were significantly higher than those in all the groups with 740 and $370 \mathrm{mg}$ of iodine/bird $(p<0.0046)$.

Inter-observer agreement for subjective image-quality assessment of six arteries with nine CM injection protocols are listed in Table 4. The $\rho$ value was calculated to confirm inter-observer agreement, which is appropriate for ordered categorical judgments [27]. Correlations between two observers for subjective evaluation of image quality were "moderate" to "strong" in the 370 and $222 \mathrm{mg}$ of iodine/bird groups. The $\rho$ value was extremely low in the $740 \mathrm{mg}$ of iodine/bird with $0.3 \mathrm{~mL} / \mathrm{s}$ group because the distribution of scores were symmetrically imbalanced [28], rending this agreement measurement meaningless. Therefore, the percentage agreement was analyzed, which resulted in averages of $97.2 \%, 92.2 \%$, and $61.7 \%$ for groups of 740,370 , and 222 mg of iodine/bird, respectively.

\section{Time-enhancement curves}

Of 90 scans, the time to reach start-scanning cutoff attenuation ( $100 \mathrm{HU})$ was similar ( $<5 \mathrm{~s}$; which is considered short). A higher frequency of CM heterogeneity was seen in groups with an IR of $0.3 \mathrm{~mL} / \mathrm{s}$ regardless of $\mathrm{CM}$ dose. To quantify $\mathrm{CM}$ heterogeneity, subjective scoring for the time-enhancement curve was performed among the nine groups (Table 5). A fixed injection dose of $740 \mathrm{mg}$ of iodine/bird and $370 \mathrm{mg}$ of iodine/bird and a faster IR led to a lower score, which reduced the risk of CM heterogeneity, but this was not significant $(p>0.05)$. Two-way ANOVA showed that the IR affected the results significantly $(p=0.0189)$.

\section{Discussion}

By reducing the $\mathrm{CM}$ injection dose, the attenuation of major arteries decreased. By increasing the IR of $\mathrm{CM}$, the heterogeneity of $\mathrm{CM}$ distribution was limited, but this did not lead to better image quality.

Attenuation values of the AGPs in our study (600-800 HU) were higher than those of a previous study (300-500 HU) [24] using the same CM protocol (i.e., injection with $2 \mathrm{~mL}$ iopamidol followed by $0.4 \mathrm{~mL}$ of saline, with an IR of $0.3 \mathrm{~mL} / \mathrm{s}$ ). That result may have been achieved using an optimal delay and precise timing for CT. In our study, scanning was triggered manually once a region of interest (Rol) attenuation of $100 \mathrm{HU}$ was reached; in the previous study, scanning was triggered manually when peak enhancement was reached. The lower attenuation values of the previous study might have been owing to the late scanning-starting time, which may have been too late for timing of highly enhanced attenuation.

The minimal enhancement level required for diagnostic CTA for avian arteries is unknown. Diameters of the major arteries of AGPs are similar to those of human coronary arteries (about 2-4 mm) [29], so the regular attenuation of coronary arteries in HM were used as a reference. Optimal attenuation for human 
vessels is controversial [30]. In humans, atherosclerotic plaques can be calcified or non-calcified. Mean attenuation for calcified plaques is $162-820 \mathrm{HU}$, and that for non-calcified plaques is 14-125 HU [31]. To distinguish plaques from vessel lumina, optimal vessel attenuation of 250-300 HU has been reported and enhancement $>350 \mathrm{HU}$ may interfere with arterial calcifications [30]. Some scholars postulate that optimal attenuation should be higher for these small arteries and ideally lie at 325-500 HU, and that attenuation $>500 \mathrm{HU}$ is unnecessarily high and leads to an underestimation of coronary calcifications [22, 3234]. CTA accuracy is affected considerably by vessel attenuation. One study showed that greater attenuation could allow better depiction of vessel lumina, leading to higher diagnostic accuracy [35]. Inadequate attenuation could cause blurring of vessel margins and greater risks of incorrect diagnoses [35, 36]. In the $740 \mathrm{mg}$ of iodine/bird groups, attenuation was too high (> $500 \mathrm{HU}$ ). For the $370 \mathrm{mg}$ of iodine/bird groups, attenuation was mostly $325-500 \mathrm{HU}$. In the 222 $\mathrm{mg}$ of iodine/bird groups, attenuation for all the major arteries was about 250-330 HU; nevertheless, the contours of many arteries were blurred and difficult to identify.

A faster IR is believed to result in higher attenuation compared with that using a lower IR [22]. This phenomenon was not observed in our study, and might have been owing to the short duration of highly enhanced attenuation in AGPs originating from a high cardiac output. The scanning time for these major arteries might start later than the time of peak contrast enhancement, even though whole-image acquisition started immediately after signal attenuation reached $100 \mathrm{HU}$.

Image noise using a protocol of $740 \mathrm{mg}$ of iodine/bird was significantly higher than that of other study groups. Similar image noise was obtained among different IR groups. In human studies, increased image noise can limit diagnostic accuracy if evaluating small arteries [37, 38] but whether and how image noise affects diagnostic accuracy in birds is unknown. SNR and CNR were significantly higher in the 222 mg of iodine/bird groups compared with those administered $740 \mathrm{mg}$ of iodine/bird. This was because the image noise of the $740 \mathrm{mg}$ of iodine/bird groups was too high, which led to reductions in SNR and CNR.

The diameter of major arteries was influenced by changes in CM injection dose. This finding could be related to the blooming artifact caused by excessive attenuation of vessels. The blooming artifact distorts high-attenuation objects to appear larger than actuality, and may be superimposed by additional motion artifacts related to high heart rates $[39,40]$. In HM, these high-attenuation objects usually are densely calcified plaques or metallic implants (e.g., coronary stents) [39]. Arterial attenuation in our study using $370 \mathrm{mg}$ and $740 \mathrm{mg}$ of iodine/bird was higher than that of calcified plaques ( $391 \pm 156 \mathrm{HU}$ ) [31]; therefore, the diameter of arteries may be overestimated at high doses.

With respect to $740 \mathrm{mg}$ of iodine/bird groups, excessive attenuation of arteries caused streak artifacts in several images, but these artifacts did not affect the vessels excessively. Streak artifacts can occur with the appearance of high-attenuation objects, such as metallic implants or vessels filled with high iodine concentrations [39]. In our study, these artifacts were solved with lower doses of CM; other solutions include using a saline bolus flush or a low concentration of CM [39].

We documented two failures of CM injection owing to CM leakage from the intravenous catheter using an IR of $0.5 \mathrm{~mL} / \mathrm{s}$. The maximum IR of $\mathrm{CM}$ is influenced by catheter size, total injection volume, or patient size [41]. Higher IRs may result in extravasation of the intravenous CM. An IR of $0.5 \mathrm{~mL} / \mathrm{s}$ did not exceed the recommended maximum IR for CM using a $24-\mathrm{G}$ catheter $(1.0 \mathrm{~mL} / \mathrm{s}$ in children), but the size of an AGP is far smaller than that of a child. This may have been a reason for the high prevalence $(6.7 \%$ [2/30]) of injection failure for the $0.5 \mathrm{~mL} / \mathrm{s}$ groups. Our results revealed the non-necessity of IR $>0.5 \mathrm{~mL} / \mathrm{s}$.

Crackles of the respiratory system and regurgitation were noted after three CTA experiments. Crackles might be related to CM. In HM, a high osmolarity of intravenous $\mathrm{CM}$ can increase microvascular permeability, causing non-cardiogenic pulmonary edema [42]. Nausea and vomiting are common side-effects reported in humans after receiving CM [43]. However, regurgitation might have been owing to a side-effect either from CM or anesthesia.

Our study had three limitations. First, the sample size was small compared to HM studies. Second, only three injection doses were used, which limited identification of the most appropriate dose for CTA in birds. Furthermore, our study used a one-dose-fits-all approach owing to the limitation of the instrument. Taking bodyweight into consideration, using the bodyweight-based dose in larger avian patients would be rational. Third, we did not record the full timeenhancement curve. Further study is needed to justify the timing and duration of peak enhancement to understand the optimum scan timing.

\section{Conclusions}

We suggest a dose of $370 \mathrm{mg}$ of iodine/bird provides images of better quality (compared with that using $222 \mathrm{mg}$ of iodine/bird), optimal attenuation, lower image noise, higher SNR, and CNR (compared with $740 \mathrm{mg}$ of iodine/bird). We also suggest an IR of $0.4 \mathrm{~mL} / \mathrm{s}$ or $0.5 \mathrm{~mL} / \mathrm{s}$ to improve the heterogeneity of contrast enhancement. Therefore, we recommend a combination of $370 \mathrm{mg}$ of iodine/bird with $0.4 \mathrm{~mL} / \mathrm{s}$ for clinical use if complications and injection failures are taken into account.

\section{Methods}

\section{Ethical approval of the study protocol}

The study protocol was approved (NTU107-EL-00001) by the Institutional Animal Care and Use Committee of National Taiwan University. The number of animal used was minimum determined based on previous reports with similar animal species and study designs. This manuscript was prepared in compliance with the ARRIVE guidelines for reporting animal in vivo experiments.

Ten adult AGPs (four males and six females; $3-8 \mathrm{y}$ ) were obtained from commercial breeders/pet shops. The mean bodyweight was $454.4 \pm 50.4 \mathrm{~g}$ (range, $361-530.5 \mathrm{~g})$. 
AGPs were housed in individual stainless-steel cages $(60 \times 62 \times 87 \mathrm{~cm})$ in a well-ventilated room $(4 \times 4 \times 5 \mathrm{~m})$ at $25-30^{\circ} \mathrm{C}$. Each cage contained environment enrichment toys. An opportunity to exercise and socialize in the room was provided once weekly.

AGPs were offered a commercial pelleted diet (Nutribird P19 Tropical and Nutribird G18 Tropical; Versele-Laga, Deinze, Belgium) with one teaspoon of mixed grains, seeds, and nuts (Vitpower parrot and macaw food; Wanfeng, Taipei, Taiwan) daily and were offered tap water ad libitum from a stainless-steel water bowl. AGPs had lived at the facility for $\geq 2 \mathrm{y}$ before experimentation.

Regular physical examination, blood examination, and whole-body radiographs were obtained. A physical examination involving auscultation of the lungs and heart was performed to ensure each AGP was healthy with clear lung sounds, regular heart rhythm, and no heart murmur. Blood examinations comprised manual complete blood count (packed cell volume, and counts of red blood cells, white blood cells [WBCs] and WBC differential counts), serum biochemistry (aspartate aminotransferase, blood urea nitrogen, calcium, cholesterol, creatine kinase, gamma-glutamyl transferase, glucose, lactate dehydrogenase, phosphorus, total protein, triglycerides, uric acid, and electrolytes) using a VITROS ${ }^{\circledR} 350$ system (Ortho Clinical Diagnostics, Johnson \& Johnson, Melbourne, Australia), and protein electrophoresis (SPIFE ${ }^{\circledR} 3000$; Helena Laboratories, Beaumont, TX, USA). Radiography (KXO-32 s; Toshiba, Tokyo, Japan) included ventrodorsal and right lateral views.

Additional examinations for cardiological evaluation comprised five-lead electrocardiography (AT-1 ${ }^{\circledR}$ Smartprint; Schiller, Baar, Switzerland) [44], echocardiography using a ventral midline approach (EnVisor ${ }^{\circledR}$ HD with S12 UltraBand Sector Xducer; Philips Electronics North America, Andover, MA, USA) [45], and polymerase chain reaction for specific pathogens associated with cardiac diseases (i.e., polyomavirus, Chlamydia psittaci, and bornavirus). These examinations and the taking of diagnostic samples were performed under anesthesia. AGPs were included only if they were considered healthy following these examinations [46].

\section{Study design}

Three IRs of CM (0.3 [used previously], $0.4,0.5 \mathrm{~mL} / \mathrm{s})$ combined with three test doses of CM (740 mg of iodine/bird [2 mL, used previously], $370 \mathrm{mg}$ of iodine/bird [1 mL], $222 \mathrm{mg}$ of iodine/bird [0.6 mL]) (lopamiro $370^{\circledR}$; Bracco s.p.a., Milan, Italy) were tested [23,24]. Therefore, there were nine treatment groups. This study had a prospective, crossover design with a washout period of $\geq 1$ month between protocols. If artifacts were present on the time-enhancement curve, or other reasons caused imaging failure, a second scan with that specific protocol was undertaken after a washout period.

\section{Anesthesia protocol}

CT was undertaken with AGPs under general anesthesia. AGPs were fasted for $4-6 \mathrm{~h}$ before anesthesia, induced by $5 \%$ isoflurane (Attane ${ }^{\circledR}$; Panion \& BF Biotech, Taoyuan, Taiwan) and oxygen ( $2 \mathrm{~L} / \mathrm{min})$, in an induction chamber. Then, each AGP was intubated with a 3.0-mm endotracheal tube (Jorgensen Laboratories, Loveland, CO, USA) and a 24-G catheter (Surflo ${ }^{\circledR} 24 \mathrm{G} \times 3 / 4$ "; Terumo, Biñan, Philippines) was placed in the basilic vein.

A veterinary anesthesia-delivery system (ADS $2000^{\circledR}$; Engler, Hialeah, FL, USA) was connected and used to maintain anesthesia with $3-5 \%$ isoflurane depending on the bird's depth of anesthesia, which was evaluated by pulse oximetry and a $\mathrm{CO}_{2}$ detector ( $9847 \mathrm{~V}$; Nonin Medical, Plymouth, MN, USA), auscultation, involuntary corneal reflex, and muscle tone. Machine settings were: flow rate, $0.8 \mathrm{~L} / \mathrm{min}$; breaths, $17-18$ times/min; peak inspiratory pressure (PIP), 7-8 mmHg. Supplemental heat was provided by a heating lamp.

To diminish motion artifacts and enable data acquisition, the anesthesia protocol was adjusted once the contrast study was initiated, as follows: $4-5 \%$ isoflurane depending on the anesthesia depth; flow rate, $0.6 \mathrm{~mL} / \mathrm{min}$; breaths, 2 times/min; PIP, $5 \mathrm{mmHg}$.

Following completion of diagnostic imaging, the intravenous catheter was removed, and the final physical examination was performed to identify related sideeffects. Lactated Ringer's solution ( $25 \mathrm{~mL}$, subcutaneous) was administered to reduce the potential adverse renal effects of $\mathrm{CM}$. After the procedures, the overall conditions, appetite, fecal output, responsiveness, and behavior were monitored for the following three days.

\section{CTA protocol}

CM was delivered by a dual-head power injector system (OptiVantage ${ }^{\circledR}$; Guerbet, France).

AGPs were positioned in dorsal recumbency on a V-shaped trough during imaging using a 16-detector row CT (Activion 16; Toshiba, Tokyo, Japan). Wholebody "scout" scans were obtained first (cranial-to-caudal) to map the longitudinal field of view of the helical scan. According to the scout image, the scan range was determined from the last cervical vertebrae to the coxofemoral joint. To located the AA, pre-contrast helical scans were obtained in slice thickness (section collimation) of $0.5 \mathrm{~mm}$, and section width of $3 \mathrm{~mm}$, from cranial-to-caudal, with a peak electric potential of $120 \mathrm{kVp}$, electrical current of $50 \mathrm{~mA}$, helical pitch of 1.0 , rotation speed of $0.5 \mathrm{~s}$, and table feed of $10 \mathrm{~mm} / \mathrm{s}$. The matrix size was $512 \times 512$ pixels.

The AA path was determined by reference to unenhanced images. During the contrast study, identical scanning parameters were used, and a dynamic scan was undertaken simultaneously using a circular Rol in the middle portion of the AA to monitor attenuation values in time-enhancement curves.

Synchronization between CM administration by the injector system and data acquisition was achieved with a real-time bolus-tracking method, and the scan was triggered manually once enhancement reached $100 \mathrm{HU}[23,24]$.

\section{Objective (quantitative) image quality}

Parameters of quantitative image quality, including attenuation, image noise, SNR, and CNR, were measured in six major arteries (middle segment of left brachiocephalic trunks; middle segment of right brachiocephalic trunks; AA root; middle segment of the left pulmonary artery; middle segment of the right 
pulmonary artery; middle segment of abdominal aorta) for each image. Artery diameters were also measured. Cross-sectional images were magnified to $800 \%$ to improve measurement accuracy.

To obtain parameters, a circular Rol was placed in each artery by one observer and was enlarged to include the entire vascular lumen; the Rol size was recorded. Attenuation of the six targeted arteries was obtained as mean HU by placing the Rol in each vessel. "Image noise" was defined as the standard deviation (SD) of attenuation (in $\mathrm{HU}$ ) in an Rol in targeted arteries. Attenuation of the right rhomboideus superficialis muscle was measured at the level of the artery measured using a Rol of area $0.2 \mathrm{~cm}^{2}$. To minimize bias from use of a single measurement, measurements of attenuation of arteries, image noise, and attenuation of the rhomboideus superficialis muscle were repeated thrice and averaged. These values were used for calculation of SNR, CNR, and diameter.

SNR was defined as mean attenuation (HU) divided by image noise, and calculated using the formula:

$$
S N R=\frac{\text { Mean Rol attenuation value }}{\text { Image noise }}
$$

In HM, CNR is usually defined as the difference between the mean attenuation (HU) of vessels and perivascular fat or muscle divided by image noise. Owing to a lack of perivascular tissue in birds, we chose the rhomboideus superficialis muscle and used the formula:

$$
C N R=\frac{\text { Mean } \text { Rol attenuation value }- \text { mean muscle attenuation value }}{\text { lmage noise }}
$$

Arterial diameter (D) was calculated manually:

$$
D=2 \times\left(\frac{\text { size of } R o l}{\pi}\right)^{1 / 2}
$$

Two observers participated in Rol measurements. Observer 1 (WWL) obtained all measurements twice to evaluate intra-observer agreement. Observer 2 (YPH) obtained measurements once to evaluate inter-observer agreement.

\section{Subjective (qualitative) image quality}

Two observers evaluated image quality independently, and were blinded to the protocol. "Image quality" was defined by: vascular enhancement, sharpness of the artery contour, artifacts, and diagnostic ability. On the basis of this definition, each reader graded the image quality of the six major arteries independently on a four-point scale (Table 6). In the case of discordant scores between two observers, the higher score was used.

\section{Timeenhancement curves}

The time-enhancement curve of each scan was recorded using a smartphone camera upon CM injection. An observer analyzed 90 time-enhancement curves while blinded to the protocol. $\mathrm{CM}$ heterogeneity was evaluated in each curve and a qualitative scale from 0 to $2(0=$ absence of $\mathrm{CM}$ heterogeneity; $1=$ mild heterogeneity; 2 = severe heterogeneity) was scored.

\section{Statistical analyses}

Statistical analyses were undertaken using SPSS 21.0 (IBM, Armonk, NY, USA), Prism 7.04 (GraphPad, San Diego, CA, USA), and Excel ${ }^{\mathrm{TM}} 2013$ (Microsoft, Redmond, WA, USA). For quantitative variables, normally distributed data are shown as mean \pm SD (i.e., $95 \%$ confidence intervals (Cls) of the mean). Nonnormally distributed variables are expressed as the median with 2.5 th and 97.5 th percentiles (i.e., $95 \%$ Cls of the median). Normality was assessed by the Shapiro-Wilk test. Categorical variables are expressed as frequencies (percentages).

Two-way analysis of variance (ANOVA) was conducted to test for differences between the nine treatment groups with normally distributed data, whereas differences were calculated through the Kruskal-Wallis test for non-normally distributed data. The alpha level for all tests was $p=0.05$. Correction for multiple comparisons was performed using Tukey's multiple comparisons test or Dunn's multiple-comparisons test.

Agreements between attenuation and diameter were explored using Spearman's correlation coefficient ( $\rho$ ), and differences were investigated with ANOVA setting a cutoff of $p<0.05$. $\rho$ - values were categorized as follows: $<0-$ no agreement; 0 to 0.20 -very weak agreement; 0.21 to $0.40-$ weak agreement; 0.41 to 0.60 -moderate agreement; 0.61 to 0.80 -strong agreement; 0.81 to 1 -very strong agreement [47].

\section{Abbreviations}

CM: contrast medium; CTA: Computed tomography angiography; HM: human medicine; IR: Injection rate; HU: Hounsfield unit; AA: Ascending aorta; SNR: Signal-to-noise ratio; CNR: Contrast-to-noise ratio; Rol: Region of interest; PIP: Peak inspiratory pressure; SD: Standard deviation; D: Arterial diameter; Cls: Confidence intervals

\section{Declarations}

\section{Ethics approval and consent to participate}


The study protocol was approved (NTU107-EL-00001) by the Institutional Animal Care and Use Committee of National Taiwan University. Authors adhered to ARRIVE guidelines.

\section{Consent for publication}

Not applicable.

\section{Availability of data and materials}

The datasets used and/or analyzed during the current study are available from the corresponding author on reasonable request.

\section{Competing interests}

The authors declare that they have no competing interests.

\section{Funding}

This work was supported by the Ministry of Science and Technology (Taiwan) [107-2311-B-002-005-]. The funding body did not play a role in the design, analysis and reporting of the study.

\section{Authors' contributions}

Conception and design: WL. Wang and PH. Yu. Data acquisition: WL. Wang, FL. Chang, and PH. Yu. Data analysis/interpretation: WL. Wang. Writing-original draft: WL. Wang. Writing-review and editing: WL. Wang and PH. Yu. All authors have read and approved the final manuscript.

\section{Acknowledgements}

We acknowledge Ai-Ting Lin's professional technical support with CTA. This work was supported by the Ministry of Science and Technology (Taiwan) [1072311-B-002-005-].

\section{Authors' information}

ORCID

Pin-Huan Yu https://orcid.org/0000-0002-3788-030X

Wen-Lin Wang https://orcid.org/0000-0002-3360-0649

\section{References}

1. Johnson JH, Phalen DN, Kondik VH, et al. Atherosclerosis in psittacine birds. Proc Annu Conf Assoc Avian Vet 1992;87-93.

2. Beaufrère $\mathrm{H}$. Characterization, prevalence, and risk factors of spontaneous and experimental atherosclerosis and vascular imaging in psittaciformes [Dissertation]. Baton Rouge, Louisiana: Louisiana State University; 2013.

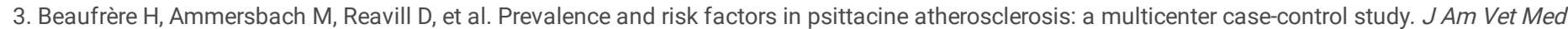
Assoc 2013;242(12):1696-1704.

4. Dorrestein G, Zwart P, Borst G, et al. Causes of disease and death in birds. Tijdschr Diergeneeskd 1977;102(7):437-447.

5. Finlayson R, Symons C, Tw-Fiennes R. Atherosclerosis: a comparative study. BMJ 1962;1(5277):501.

6. Fox H. Arteriosclerosis in lower mammals and birds: its relation to the disease in man. Arteriosclerosis 1933;153-193.

7. Grunberg W. Spontaneous arteriosclerosis in birds. Bull Soc R Zool Anvers 1964;43:479-488.

8. Kellin N. Auswertung der Sektions-und Laborbefunde von 1780 Vögeln der Ordnung Psittaciformes in einem Zeitraum von vier Jahren (2000 bis 2003 ) [Dissertation]. Giessen, Germany: University of Giessen, VVB Laufersweiler Verlag; 2009.

9. Pilny A. Retrospective of atherosclerosis in psittacine birds: clinical and histopathologic findings in 31 cases. Proc Annu Conf Assoc Avian Vet 2004:349351.

10. Abou-Zahr T, Dutton TAG, Carrasco DC, et al. Atherosclerotic aortic aneurysm in a steppe eagle (Aquila nipalensis). Vet Record Case Rep 2015;3(1):e000218.

11. Beaufrère $\mathrm{H}$, Holder KA, Bauer R, et al. Intermittent claudication-like syndrome secondary to atherosclerosis in a yellow-naped Amazon parrot (Amazona ochrocephala auropalliata). J Avian Med Surg 2011;25(4):266-276.

12. Facon $\mathrm{C}$, Beaufrère $\mathrm{H}$, Gaborit $\mathrm{C}$, et al. Cluster of atherosclerosis in a captive population of black kites (Milvus migrans subsp.) in France and effect of nutrition on the plasma lipid profile. Avian Dis 2014;58(1):176-182.

13. Mans C, Brown CJ. Radiographic evidence of atherosclerosis of the descending aorta in a grey-cheeked parakeet (Brotogeris pyrrhopterus). J Avian Med Surg 2007;21(1):56-62.

14. Phalen DN, Hays HB, Filippich LJ, et al. Heart failure in a macaw with atherosclerosis of the aorta and brachiocephalic arteries. J Am Vet Med Assoc 1996;209(8):1435-1440. 
15. Sedacca CD, Campbell TW, Bright JM, et al. Chronic cor pulmonale secondary to pulmonary atherosclerosis in an African Grey parrot. J Am Vet Med Assoc 2009;234(8):1055-1059.

16. Shrubsole-Cockwill A, Wojnarowicz C, Parker D. Atherosclerosis and ischemic cardiomyopathy in a captive, adult red-tailed hawk (Buteo jamaicensis). Avian Dis 2008;52(3):537-539.

17. Beaufrère $\mathrm{H}$, Nevarez $\mathrm{J}$, Gaschen $\mathrm{L}$, et al. Diagnosis of presumed acute ischemic stroke and associated seizure management in a Congo African grey parrot. J Am Vet Med Assoc 2011;239(1):122-128.

18. Grosset C, Guzman DSM, Keating MK, et al. Central vestibular disease in a blue and gold macaw (Ara ararauna) with cerebral infarction and hemorrhage. J Avian Med Surg 2014;28(2):132-142.

19. Oster SC, Jung S, Moon R. Resolution of supraventricular arrhythmia using sotalol in an adult golden eagle (Aquila chrysaetos) with presumed atherosclerosis. J Exotic Pet Med 2019;29:136-141.

20. Fernandez-Friera L, Ibanez B, Fuster V. Imaging subclinical atherosclerosis: is it ready for prime time? A review. J Cardiovasc Trans/ Res 2014;7(7):623634.

21. Leipsic J, Abbara S, Achenbach S, et al. SCCT guidelines for the interpretation and reporting of coronary CT angiography: a report of the Society of Cardiovascular Computed Tomography Guidelines Committee. J Cardiovasc Comput Tomogr 2014;8(5):342-358.

22. Bae KT. Intravenous contrast medium administration and scan timing at CT: considerations and approaches. Radiology 2010;256(1):32-61.

23. Lee YL, Yu PH, Chen CL, et al. Determination of the enhancement effect and diameters of the major arteries of African grey parrots using a dual-head power injector for computed tomographic angiography. Taiwan Vet $J$ 2015;41(3):165-175.

24. Yu PH, Lee YL, Chen CL, et al. Comparison of three computed tomographic angiography protocols to assess diameters of major arteries in African grey parrots (Psittacus erithacus). Am J Vet Res 2018;79(1):42-53.

25. Beaufrère $H$, Rodriguez $D$, Pariaut R, et al. Estimation of intrathoracic arterial diameter by means of computed tomographic angiography in Hispaniolan Amazon parrots. Am J Vet Res 2011;72(2):210-218.

26. Krautwald-Junghanns ME, Schloemer J, Pees M. lodine-based contrast media in avian medicine. J Exotic Pet Med 2008;17(3):189-197.

27. Gwet K. Handbook of Inter-Rater Reliability: The Definitive Guide to Measuring the Extent of Agreement Among Raters. Gaithersburg: Advanced Analytics, 2010.

28. Shankar V, Bangdiwala SI. Observer agreement paradoxes in 2x2 tables: comparison of agreement measures. BMC Med Res Methodo/2014;14:100.

29. Dodge Jr JT, Brown BG, Bolson EL, et al. Lumen diameter of normal human coronary arteries. Influence of age, sex, anatomic variation, and left ventricular hypertrophy or dilation. Circulation 1992;86(1):232-246.

30. Becker CR, Hong C, Knez A, et al. Optimal contrast application for cardiac 4-detector-row computed tomography. Investig Radio/ 2003;38(11):690-694.

31. Leber AW, Knez A, Becker A, et al. Accuracy of multidetector spiral computed tomography in identifying and differentiating the composition of coronary atherosclerotic plaques: a comparative study with intracoronary ultrasound. J Am Coll Cardio/ 2004;43(7):1241-1247.

32. Eijsvoogel NG, Hendriks BMF, Nelemans P, et al. Personalization of CM injection protocols in coronary computed tomographic angiography (People CT Trial). Contrast Media Mol Imaging 2020;2020:5407936.

33. Fei X, Du X, Yang Q, et al. 64-MDCT coronary angiography: phantom study of effects of vascular attenuation on detection of coronary stenosis. Am J Roentgenol 2008;191(1):43-49.

34. Isogai T, Jinzaki M, Tanami Y, et al. Body weight-tailored contrast material injection protocol for 64-detector row computed tomography coronary angiography. Jap J Radio/ 2011.29(1):33-38.

35. Cademartiri F, Mollet NR, Lemos PA, et al. Higher intracoronary attenuation improves diagnostic accuracy in MDCT coronary angiography. Am $J$ Roentgenol 2006;187(4):W430-W433.

36. Yan RT, Miller JM, Rochitte CE, et al. Predictors of inaccurate coronary arterial stenosis assessment by CT angiography. JACC Cardiovasc Imaging 2013.6(9):963-972.

37. Alkadhi H, Scheffel H, Desbiolles L, et al. Dual-source computed tomography coronary angiography: influence of obesity, calcium load, and heart rate on diagnostic accuracy. Eur Heart J 2008;29(6):766-776.

38. MacKenzie JD, Nazario-Larrieu J, Cai T, et al. Reduced-dose CT: effect on reader evaluation in detection of pulmonary embolism. Am J Roentgenol 2007;189(6):1371-1379.

39. Ghekiere O, Salgado R, Buls N, et al. Image quality in coronary CT angiography: challenges and technical solutions. Br J Radiol 2017;90(1072):20160567.

40. Scheffel H, Alkadhi H, Plass A, et al. Accuracy of dual-source CT coronary angiography: First experience in a high pre-test probability population without heart rate control. Eur Radiol 2006.16(12):2739-2747.

41. Callahan MJ, Servaes S, Lee EY, et al. Practice patterns for the use of iodinated i.v. contrast media for pediatric CT studies: a survey of the Society for Pediatric Radiology. Am J Roentgenol 2014.202(4):872-879.

42. Paul RE, George G. Fatal non-cardiogenic pulmonary oedema after intravenous nonionic radiographic contrast. Lancet 2002;359(9311):1037-1038.

43. Katayama H, Yamaguchi K, Kozuka T, et al. Adverse reactions to ionic and nonionic contrast media. A report from the Japanese Committee on the Safety of Contrast Media. Radiology 1990;175(3):621-628.

44. Lumeij JT, Ritchie BW. Chapter 27 - Cardiology. In: Ritchie BW, Harrison GJ, Harrison LR, eds. Avian Medicine: Principles and Application. Florida: Wingers Publishing, 1994: 695-722. 
45. Krautwald-Junghanns ME, Schulz M, Hagner D, et al. Transcoelomic two-dimensional echocardiography in the avian patient. $J$ Avian Med Surg 1995;9(1):19-31.

46. Hawkins MG, Guzman DSM, Beaufrère H, et al. Chapter 5 - Birds. In: Carpenter JW, Marion CJ, eds. Exotic Animal Formulary, Fifth Edition. Missouri: Elsevier, 2018: 167-375.

47. Evans JD. Straightforward statistics for the behavioral sciences. Pacific Grove: Brooks/Cole Publishing, 1996.

\section{Tables}

Table 1. Inter-observer agreement of measured parameters at six major arteries in the nine test groups.

$740 \mathrm{mg}$ of iodine/bird
$0.3 \mathrm{~mL} / \mathrm{s} \quad 0.4 \mathrm{~mL} / \mathrm{s}$

$370 \mathrm{mg}$ of iodine/bird

$222 \mathrm{mg}$ of iodine/bird

-: Calculation of the correlation coefficient could not be performed because all 10 measured values were similar.

Table 2. Intra-observer agreement (reproducibility) of measured parameters at six major arteries in the nine test groups.

$740 \mathrm{mg}$ of iodine/bird
$370 \mathrm{mg}$ of iodine/bird

$0.5 \mathrm{~mL} / \mathrm{s}$

$0.3 \mathrm{~mL} / \mathrm{s} \quad 0.4 \mathrm{~mL} / \mathrm{s}$
$0.3 \mathrm{~mL} / \mathrm{s} \quad 0.4 \mathrm{~mL} / \mathrm{s}$

$0.4 \mathrm{~mL} / \mathrm{s} \quad 0.5 \mathrm{~mL} / \mathrm{s}$

\section{Attenuation}

Left brachiocephalic trunks

Right brachiocephalic trunks

Ascending aorta

Left pulmonary artery

Right pulmonary artery

Abdominal aorta

Diameter

0.976

0.988

0.842

0.976

0.855

0.988

0.891

.988

0.988

0.988

0.952

0.988

0.976

0.952

0.952

0.855

0.976

0.9
0.9
0.9
0.
0.9
0.9

0.988

0.964

1.000

0.988

$0.988 \quad 0.988$

$0.794 \quad 0.98$

0.952

0.927

0.952

0.976

0.988
0.9
0.988
0.9
0.8
0.9

0.988

0.976

0.988

0.988

0.875

0.976

0.952

1.000

$\begin{array}{llll}0.952 & 0.964 & 0.988 & 0.952\end{array}$

1.000

$\begin{array}{llll}0.952 & 0.964 & 0.988 & 0.952\end{array}$

1.000

Left brachiocephalic trunks

Right brachiocephalic trunks

Ascending aorta

0.941

0.923

0.953

0.975

0.969

0.988

0.927

0.988

0.90

0.988

0.949

0.783

0.965

0.969

0.997

0.994

1.000

0.981

0.947

$0.920 \quad 0.854$

0.801

0.951

0.988

0.997

0.969

0.943

0.962

0.975

0.959

0.982

Left pulmonary artery

0.900

0.912

0.690

0.939

0.797

0.902

Right pulmonary artery

0.864

Abdominal aorta

0.509

0.688

$0.869 \quad 1.000$

$0.764 \quad 0.995$

0.976

$1.000 \quad 0.583$

0.767

1.000

1.000

1.000

0.867

$0.817 \quad 0.802$


-: Calculation of the correlation coefficient could not be done because all 10 measured values were similar.

Table 3. Comparison of subjective image quality between nine groups.

\begin{tabular}{|c|c|c|c|c|c|c|c|c|c|c|c|c|c|c|c|c|c|c|c|c|c|c|c|c|c|c|c|}
\hline & & LBC & & & & & & & & & endi & g A & & & PA & & & & t PA & & & & omin & A & & Total [ & (\%) \\
\hline & Score & 1 & 2 & 3 & 4 & 1 & 2 & 3 & 4 & 1 & 2 & 3 & 4 & 1 & 2 & 3 & 4 & 1 & 2 & 3 & 4 & 1 & 2 & 3 & 4 & 1 & 2 \\
\hline \multirow[t]{3}{*}{$\begin{array}{l}740 \mathrm{mg} \text { of } \\
\text { iodine/bird }\end{array}$} & $\begin{array}{l}0.3 \\
\mathrm{~mL} / \mathrm{s}\end{array}$ & 0 & 10 & 0 & 0 & 0 & 10 & 0 & 0 & 0 & 10 & 0 & 0 & 0 & 10 & 0 & 0 & 0 & 10 & 0 & 0 & 0 & 10 & 0 & 0 & $0(0)$ & $\begin{array}{l}6 \mathrm{C} \\
(1\end{array}$ \\
\hline & $\begin{array}{l}0.4 \\
\mathrm{~mL} / \mathrm{s}\end{array}$ & 0 & 10 & 0 & 0 & 0 & 10 & 0 & 0 & 0 & 10 & 0 & 0 & 1 & 9 & 0 & 0 & 0 & 10 & 0 & 0 & 0 & 10 & 0 & 0 & $\begin{array}{l}1 \\
(1.7)\end{array}$ & $\begin{array}{l}5 c \\
(9\end{array}$ \\
\hline & $\begin{array}{l}0.5 \\
\mathrm{~mL} / \mathrm{s}\end{array}$ & 0 & 10 & 0 & 0 & 0 & 10 & 0 & 0 & 0 & 10 & 0 & 0 & 0 & 10 & 0 & 0 & 0 & 10 & 0 & 0 & 0 & 10 & 0 & 0 & $0(0)$ & $\begin{array}{l}6 \mathrm{C} \\
(11\end{array}$ \\
\hline \multirow[t]{3}{*}{$\begin{array}{l}370 \mathrm{mg} \text { of } \\
\text { iodine/bird }\end{array}$} & $\begin{array}{l}0.3 \\
\mathrm{~mL} / \mathrm{s}\end{array}$ & 7 & 3 & 0 & 0 & 9 & 1 & 0 & 0 & 9 & 1 & 0 & 0 & 9 & 1 & 0 & 0 & 8 & 1 & 1 & 0 & 9 & 1 & 0 & 0 & $\begin{array}{l}51 \\
(85)\end{array}$ & $\begin{array}{l}8 \\
(1\end{array}$ \\
\hline & $\begin{array}{l}0.4 \\
\mathrm{~mL} / \mathrm{s}\end{array}$ & 10 & 0 & 0 & 0 & 9 & 1 & 0 & 0 & 8 & 2 & 0 & 0 & 6 & 2 & 2 & 0 & 5 & 2 & 3 & 0 & 8 & 2 & 0 & 0 & $\begin{array}{l}46 \\
(76.7)\end{array}$ & 91 \\
\hline & $\begin{array}{l}0.5 \\
\mathrm{~mL} / \mathrm{s}\end{array}$ & 6 & 3 & 1 & 0 & 7 & 3 & 0 & 0 & 5 & 4 & 1 & 0 & 8 & 1 & 1 & 0 & 8 & 1 & 1 & 0 & 8 & 2 & 0 & 0 & $\begin{array}{l}42 \\
(70)\end{array}$ & $\begin{array}{l}14 \\
(2\end{array}$ \\
\hline \multirow[t]{3}{*}{$\begin{array}{l}222 \mathrm{mg} \text { of } \\
\text { iodine/bird }\end{array}$} & $\begin{array}{l}0.3 \\
\mathrm{~mL} / \mathrm{s}\end{array}$ & 3 & 2 & 3 & 2 & 4 & 1 & 4 & 1 & 4 & 1 & 4 & 1 & 3 & 0 & 5 & 2 & 2 & 0 & 7 & 1 & 8 & 1 & 0 & 1 & $\begin{array}{l}24 \\
(40)\end{array}$ & $\begin{array}{l}5 \\
(8\end{array}$ \\
\hline & $\begin{array}{l}0.4 \\
\mathrm{~mL} / \mathrm{s}\end{array}$ & 2 & 1 & 2 & 5 & 4 & 1 & 0 & 5 & 2 & 0 & 2 & 6 & 3 & 1 & 2 & 4 & 2 & 0 & 5 & 3 & 7 & 0 & 1 & 2 & $\begin{array}{l}20 \\
(33.3)\end{array}$ & 31 \\
\hline & $\begin{array}{l}0.5 \\
\mathrm{~mL} / \mathrm{s}\end{array}$ & 2 & 1 & 3 & 4 & 3 & 1 & 3 & 3 & 2 & 1 & 2 & 5 & 4 & 1 & 1 & 4 & 3 & 2 & 1 & 4 & 7 & 1 & 0 & 2 & $\begin{array}{l}21 \\
(35)\end{array}$ & $\begin{array}{l}7 \\
(1\end{array}$ \\
\hline
\end{tabular}

Score classification of image quality: 1 = excellent; 2 = good; 3 = poor; 4 = non-diagnostic.

LBCT, left brachiocephalic trunk; RBCT, right brachiocephalic trunk; A, aorta; PA, pulmonary aorta

Table 4. Inter-observer agreement for assessment of image quality

\begin{tabular}{llll} 
& & $\begin{array}{c}\text { Correlation } \\
\text { coefficient }\end{array}$ & $\begin{array}{c}\text { Percentage } \\
\text { agreement (\%) }\end{array}$ \\
\hline $740 \mathrm{mg}$ of iodine/bird & $0.3 \mathrm{~mL} / \mathrm{s}$ & -0.034 & 93.33 \\
\cline { 2 - 4 } & $0.4 \mathrm{~mL} / \mathrm{s}$ & 1.000 & 100.00 \\
\cline { 2 - 4 } & $0.5 \mathrm{~mL} / \mathrm{s}$ & - & 98.30 \\
\hline $370 \mathrm{mg}$ of iodine/bird & $0.3 \mathrm{~mL} / \mathrm{s}$ & 0.703 & 93.33 \\
\cline { 2 - 4 } & $0.4 \mathrm{~mL} / \mathrm{s}$ & 0.658 & 93.33 \\
\hline \multirow{2}{*}{$222 \mathrm{mg}$ of iodine/bird } & $0.5 \mathrm{~mL} / \mathrm{s}$ & 0.456 & 90.00 \\
\cline { 2 - 4 } & $0.3 \mathrm{~mL} / \mathrm{s}$ & 0.457 & 60.00 \\
\hline & $0.5 \mathrm{~mL} / \mathrm{s}$ & 0.755 & 66.67 \\
\hline
\end{tabular}

-: Calculation of the correlation coefficient could not be performed because all 60 measured values were similar.

Table 5. Comparison of subjective scoring of time-enhancement curves among nine groups 


\begin{tabular}{|c|c|c|c|c|c|}
\hline & \multicolumn{4}{|c|}{ Total [n (\%)] } & \multirow{2}{*}{$\begin{array}{l}\text { Mean } \\
\text { Score }\end{array}$} \\
\hline & Score & 0 & 1 & 2 & \\
\hline \multirow[t]{3}{*}{$740 \mathrm{mg}$ of iodine/bird } & $0.3 \mathrm{~mL} / \mathrm{s}$ & $1(10 \%)$ & $3(30 \%)$ & $6(60 \%)$ & 1.5 \\
\hline & $0.4 \mathrm{~mL} / \mathrm{s}$ & $2(20 \%)$ & $5(50 \%)$ & $3(30 \%)$ & 1.1 \\
\hline & $0.5 \mathrm{~mL} / \mathrm{s}$ & $6(60 \%)$ & $2(20 \%)$ & $2(20 \%)$ & 0.6 \\
\hline \multirow[t]{3}{*}{$370 \mathrm{mg}$ of iodine/bird } & $0.3 \mathrm{~mL} / \mathrm{s}$ & $4(40 \%)$ & $2(20 \%)$ & $4(40 \%)$ & 1.0 \\
\hline & $0.4 \mathrm{~mL} / \mathrm{s}$ & $5(50 \%)$ & $2(20 \%)$ & $3(30 \%)$ & 0.8 \\
\hline & $0.5 \mathrm{~mL} / \mathrm{s}$ & $6(60 \%)$ & $3(30 \%)$ & $1(10 \%)$ & 0.5 \\
\hline \multirow[t]{3}{*}{$222 \mathrm{mg}$ of iodine/bird } & $0.3 \mathrm{~mL} / \mathrm{s}$ & $4(40 \%)$ & $2(20 \%)$ & $4(40 \%)$ & 1.0 \\
\hline & $0.4 \mathrm{~mL} / \mathrm{s}$ & $6(60 \%)$ & $2(20 \%)$ & $2(20 \%)$ & 0.6 \\
\hline & $0.5 \mathrm{~mL} / \mathrm{s}$ & $4(40 \%)$ & $6(60 \%)$ & $0(0 \%)$ & 0.6 \\
\hline
\end{tabular}

Score classification of the time-enhancement curve: $0=$ absence of heterogeneity of contrast medium; $1=$ mild heterogeneity; $2=$ severe heterogeneity.

Table 6. Scoring scale for the image quality of CTA: descriptive reference

Score

Score 1 (Excellent) Excellent image quality. Complete absence of artifacts or blurring, which enables sufficient definition of vessel walls and excellent attenuation of vessel lumina.

Score 2 (Good) Good image quality. Minor artifacts, suboptimal attenuation of vessel lumina, vascular contour, and lumen remain easily detectable despite mild blurring.

Score 3 (Poor) Poor image quality. Moderate artifacts, suboptimal attenuation of vessel lumina, minimal-to-mild vessel discontinuity.

Score 4 (Non- Very poor image quality. Non-diagnostic or unacceptable because of poor enhancement of vessels, vessel contours are undetectable, diagnostic) or contain severe artifacts or blurring.

\section{Figures}

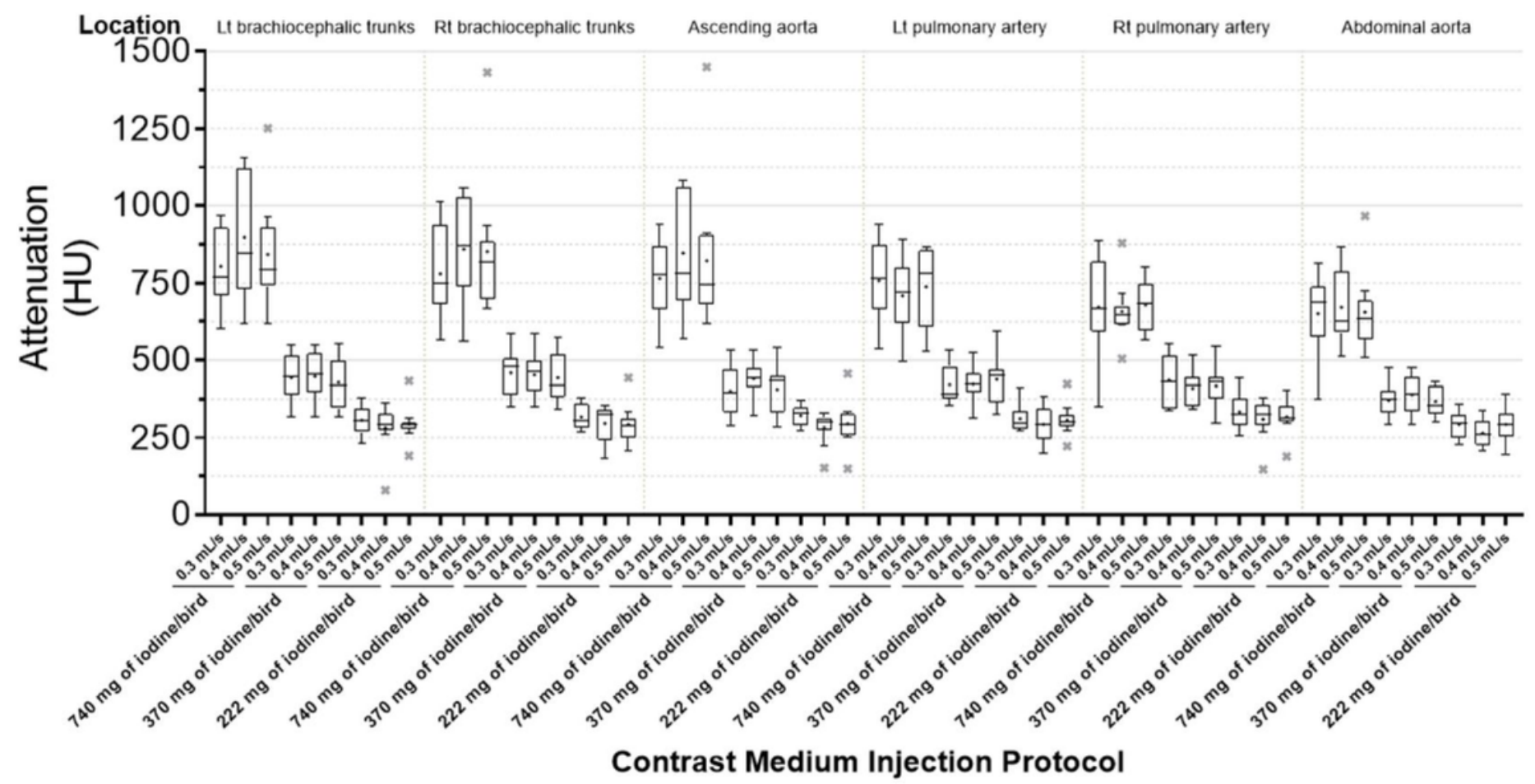


Boxplot of average attenuation (HU) within six major arteries. Vascular enhancement in the groups administered 740 mg of iodine/bird is significantly higher than that of the lowest dose groups. Attenuations with identical doses but different injection rates are nearly identical at all levels. Statistical outliers that are $>1.5$-times the interquartile range are represented by $x$.

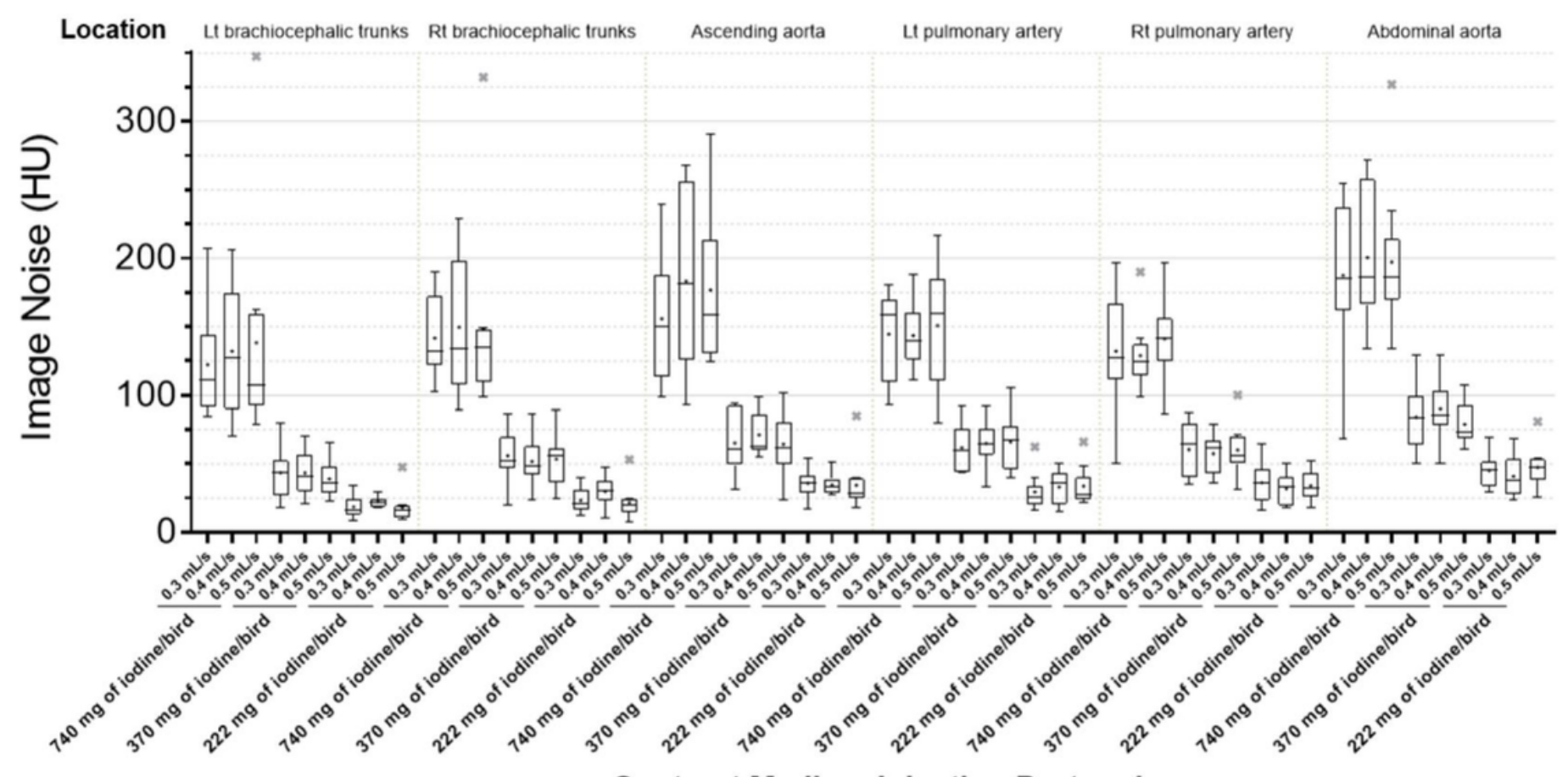

Contrast Medium Injection Protocol

\section{Figure 2}

Boxplot of image noise. As the doses of the contrast medium decrease, image noise also progressively decreases. However, with the three injection rates we tested, image noise remained similar. Statistical outliers that are $>1.5$-times the interquartile range are represented by $\times$. 

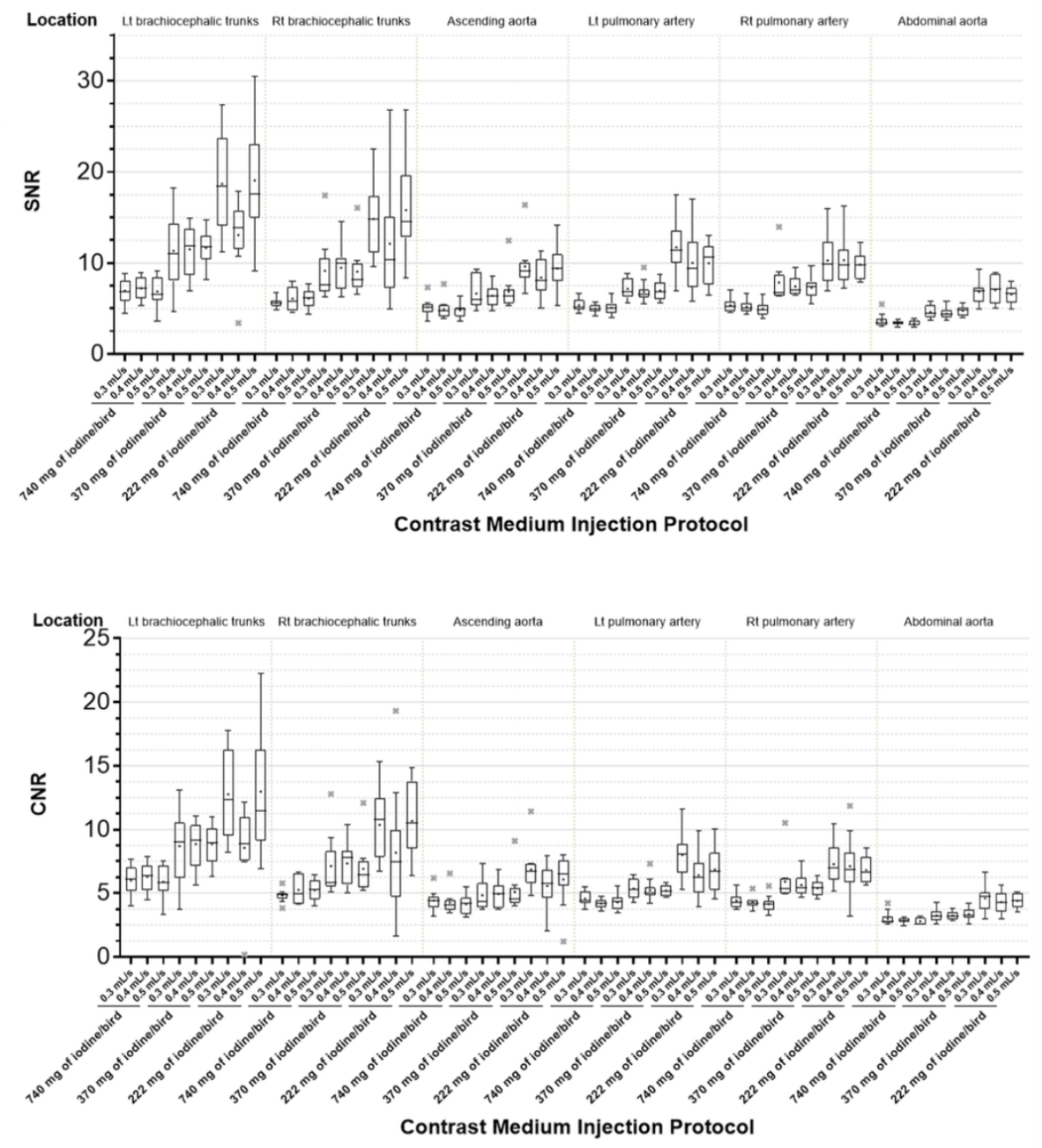

\section{Figure 3}

Boxplot of SNR and CNR. A decrease of the contrast-medium injection rate leads to a progressive improvement in SNR and CNR. However, SNR and CNR were not affected by the three injection rates we tested. Statistical outliers that are $>1.5$-times the interquartile range are represented by $x$. 


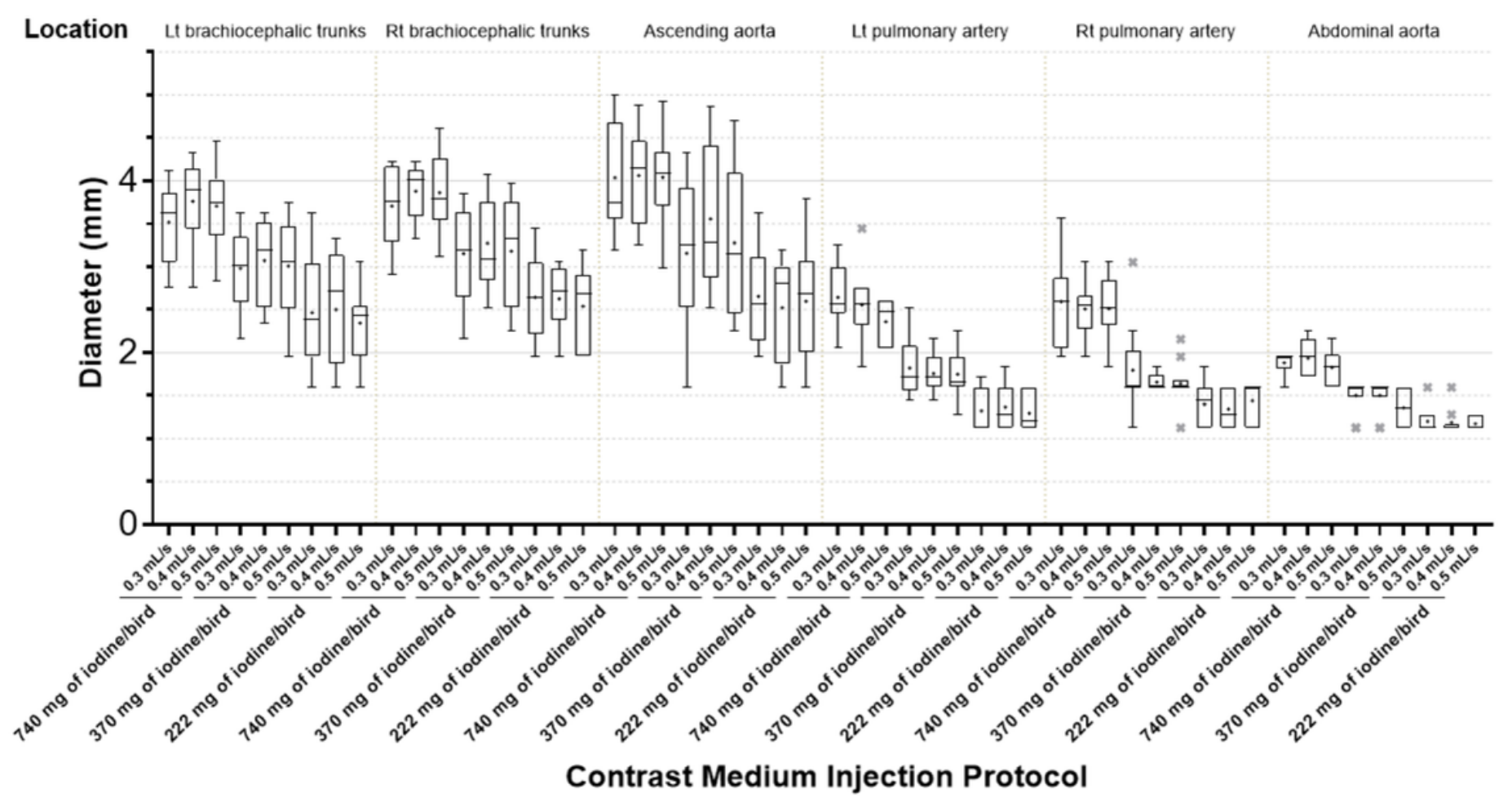

\section{Figure 4}

Boxplot of average diameter $(\mathrm{mm})$ of the six measured arteries. The measured vascular diameters are higher than those of the lower dose groups. This phenomenon is more obvious in arteries with wider diameters (i.e., left brachiocephalic trunks, right brachiocephalic trunks, ascending aorta). However, the diameters with the same dose but different injection rates are nearly identical. Statistical outliers that are $>1.5$-times the interquartile range are represented by $\mathrm{x}$. 